\title{
Prolonged Response and Restoration of Functional Independence with Bevacizumab plus Vinorelbine as Third-Line Treatment for Breast Cancer-Related Leptomeningeal Metastases
}

\author{
Emilie Le Rhun ${ }^{a, c, f}$ Sophie Taillibert ${ }^{g, h}$ Thomas Boulanger ${ }^{b}$ \\ Fahed Zairi $^{d} \quad$ Jacques Bonneterre ${ }^{a, e} \quad$ Marc C. Chamberlain ${ }^{i}$
}

${ }^{\mathrm{a}}$ Breast Cancer Department and ${ }^{\mathrm{b}}$ Department of Radiology, Oscar Lambret Center, ${ }^{c}$ Neuro-Oncology, Department of Neurosurgery, ${ }^{d}$ Department of Neurosurgery, Roger Salengro Hospital, University Hospital - CHRU, ${ }^{\mathrm{e}}$ Lille 2 North of France University, Lille, IInserm U-1192, Proteomic, Inflammatory Response, Mass Spectrometry Laboratory (PRISM), Lille 1 University, Villeneuve D'Ascq, ${ }^{9}$ Department of Neurology Mazarin and ${ }^{h}$ Department of Radiation Oncology, Pitié-Salpétrière Hospital, Paris, France; 'Department of Neurology, Fred Hutchinson Cancer Research Center, University of Washington, Seattle, Wash., USA

\section{Key Words}

Leptomeningeal metastases - Neoplastic meningitis · Breast cancer · Bevacizumab - Quality of life $\cdot$ Symptom control

\begin{abstract}
Background: Survival of patients with leptomeningeal metastases (LM) and impaired functional status is limited to several months, and rarely does neurological function improve with treatment. Case Report: A 34-year-old female with hormone-negative and HER2-positive metastatic breast cancer was diagnosed with bulky radiographic LM 45 months after initial diagnosis. She was treated with intra-CSF trastuzumab followed by intra-CSF liposomal cytarabine; however, the cancer progressed 8 months after the diagnosis of LM. At the time of the third LM progression, the patient presented with a cauda equina syndrome and cerebellar impairment resulting in an inability to walk. She was treated with CNS-directed radiotherapy (lumbosacral and cerebellar) and bevacizumab plus vinorelbine. Rapid functional improvement occurred, and the patient regained the ability to walk and independently manage
\end{abstract}

KARGER 125/s $\quad \begin{aligned} & \text { Dr. Emilie Le Rhun, MD } \\ & \text { Medical Oncology, Oscar Lambret Center } \\ & 3 \text { rue Frédéric Combemale } \\ & \text { FR-59020 Lille Cedex (France) } \\ & \text { E-Mail E-lerhun@o-lambret.fr }\end{aligned}$


LeRhun et al.: Prolonged Response and Restoration of Functional Independence with Bevacizumab plus Vinorelbine as Third-Line Treatment for Breast Cancer-Related Leptomeningeal Metastases

her daily activities. Twelve months later, she presented with rapid progression of the LM resulting in death within several weeks. Conclusion: In radiographically defined bulky LM, the combination of systemic therapy and CNS-directed radiotherapy likely is more active than intra-CSF therapy only. In lieu of the rapid and significant improvement in neurological function combined with the prolonged response, bevacizumab alone or in combination with chemotherapy and CNS-directed radiotherapy may be considered in select patients with radiographically bulky breast cancer-related $\mathrm{LM}$.

(C) 2015 S. Karger AG, Basel

\section{Background}

Breast cancer (BC) is the most common cause of solid tumor-related leptomeningeal metastases (LM) [1]. Approximately 5\% of BC patients develop LM during the course of their disease [1]. The median overall survival in BC patients with LM is limited to several months notwithstanding aggressive multimodal treatment. The neurological signs of LM are refractory to corticosteroids and rarely improve with treatment [1]. The major aim of the treatment of patients with LM is preservation of neurological function and maintenance of quality of life as LM disease progression severely compromises both neurological function and quality of life. Herein, we report on a patient with BC-related LM who exhibited dramatic functional improvement and a prolonged response to the combination of CNS-directed radiotherapy and bevacizumab plus vinorelbine.

\section{Case Report}

A 29-year-old woman was diagnosed with a 3-cm right BC. Biopsy revealed an invasive ductal carcinoma with HER2 overexpression that was negative for hormonal receptors. She initially received neoadjuvant chemotherapy [3 cycles of fluorouracil $\left(500 \mathrm{mg} / \mathrm{m}^{2}\right)$, epirubicin $\left(100 \mathrm{mg} / \mathrm{m}^{2}\right)$ and cyclophosphamide $\left(500 \mathrm{mg} / \mathrm{m}^{2}\right)$ every 3 weeks] followed by 3 cycles of docetaxel $\left(100 \mathrm{mg} / \mathrm{m}^{2}\right.$ every 3 weeks) plus trastuzumab $(6 \mathrm{mg} / \mathrm{kg}$ every 3 weeks for a total of 6 months). At the completion of chemotherapy, a radical surgery with dissection of the axillary lymph nodes was performed. No lymph node metastases or invasive residual cancer was observed; however, in situ carcinoma was seen. According to the Sataloff criteria, the therapeutic response to neoadjuvant chemotherapy was grade 2. Following surgery, external beam radiation therapy to the involved chest wall was administered. She did well until 3 years later, when hepatic, bone and lung metastases were diagnosed. The performance status of the patient remained excellent [Eastern Cooperative Oncology GroupPerformance Status (ECOG-PS) 1], and she was treated with docetaxel $\left(100 \mathrm{mg} / \mathrm{m}^{2}\right.$ every 3 weeks) plus trastuzumab (6 mg/kg every 3 weeks) for 6 months followed by trastuzumab alone as maintenance therapy.

Nine months later, she presented with progressive left deafness, headaches, nausea and gait disorder. MRI of the neuraxis revealed subarachnoid nodular (radiographically bulky disease) LM involving the cerebellum and cauda equina. CSF cytology showed suspicious tumor cells only. The systemic disease, though present, was stable. The ECOG-PS was 1, and the patient was enrolled in a phase I trial of intra-CSF trastuzumab combined with intravenous trastuzumab (6 mg/kg every 3 weeks) and oral capecitabine (2,000 $\mathrm{mg}$ twice a day for 2 weeks with 1 week off). The treatment was well tolerated, and her neurological status modestly improved. 
LeRhun et al.: Prolonged Response and Restoration of Functional Independence with Bevacizumab plus Vinorelbine as Third-Line Treatment for Breast Cancer-Related Leptomeningeal Metastases

Five months later, the patient had re-emergence of headaches associated with vomiting and blurred vision. Brain and spine MRI confirmed progression of the LM. CSF cytology again showed only signs suspicious of tumor cells. The systemic disease remained stable. The ECOG-PS had deteriorated to 2 . The patient was treated with intraventricular liposomal cytarabine (50 mg every 14 days, for a total of 4 injections), oral lapatinib (250 mg per day), intravenous trastuzumab ( $6 \mathrm{mg} / \mathrm{kg}$ every 3 weeks) and oral capecitabine $(2,000 \mathrm{mg}$ twice a day for 2 weeks with 1 week off).

Three months later, the patient sustained rapid and progressive deterioration in her neurological function accompanied by severe headaches, vomiting, diplopia and a cauda equina syndrome with urinary incontinence, sensory polyradiculopathy and lower limb weakness. As a consequence, the patient was not able to walk. Neuraxis MRI revealed worsening of both the cauda equina and cerebellum subarachnoid disease. Again, CSF cytology was negative for circulating tumor cells. Systemic disease remained stable. The ECOG-PS deteriorated to a score of 3 . Because the LM was primarily nodular and radiographically bulky and as intra-CSF chemotherapy penetrates only several millimeters into subarachnoid tumor nodules, intraventricular therapy was discontinued. Bevacizumab $(10 \mathrm{mg} / \mathrm{kg}$ every 2 weeks) in combination with vinorelbine $\left(80 \mathrm{mg} / \mathrm{m}^{2}\right)$ was initiated in conjunction with sitespecific radiotherapy (cauda equina, 30 Gy in 10 fractions; posterior fossa, 30 Gy in 10 fractions). Treatment was well tolerated, and a dramatic functional improvement was observed to systemic therapy before the onset of CNS radiation. Upon completion of radiotherapy, repeat MRI was performed and confirmed a radiographic response in both the brain and spine (fig. 1). Also, the headaches, diplopia and incontinence significantly improved, and the patient was able to resume walking without assistance and became independent in her daily activities. The patient's functional status continued to improve on treatment with a subsequent ECOG-PS of 1.

Twelve months after the third LM progression, the patient presented with rapidly progressing headaches and vomiting. Despite no significant progression of the LM on an MRI scan and continued negative CSF cytology, the patient rapidly deteriorated and died within several weeks (fig. 1).

\section{Discussion}

Currently, there are only 6 randomized clinical trials for LM; however, all have significant methodological limitations [2]. As a consequence of the paucity of data regarding treatment and management of LM, there are no standardized treatment or consensual response criteria in LM. Most often, a combination of systemic therapy, intra-CSF drug administration and CNS-directed radiotherapy are prescribed in patients with LM when clinically indicated $[1,3]$. The use of the LM-directed treatment is dictated by the general status of the patient, the underlying primary cancer, the previous treatment of the systemic disease and expected survival. Surgery, when utilized, might entail placement of a ventriculoperitoneal shunt in the case of hydrocephalus or alternatively, and more commonly, implantation of a ventricular access device to facilitate the administration of intra-CSF chemotherapy. Involved-field radiotherapy is usually administered to radiographically bulky disease, clinically symptomatic sites of disease and sites of CSF flow block as defined by radioisotope ventriculography. Nonetheless, the impact of whole-brain radiotherapy has not been clearly established in LM patients [1]. A primary cancer-specific systemic treatment is usually recommended for LM when available and the general status of the patient permits. The choice of the systemic 
LeRhun et al.: Prolonged Response and Restoration of Functional Independence with Bevacizumab plus Vinorelbine as Third-Line Treatment for Breast Cancer-Related Leptomeningeal Metastases

chemotherapy agent is based on the chemosensitivity profile of the primary tumor and also on the ability of the agent to achieve cytotoxic concentrations in CSF. However, the efficacy of intra-CSF chemotherapy in LM has not been established in prospective randomized clinical trials. The goal of intra-CSF therapy is to improve drug exposure in the CSF while reducing the risk of systemic toxicity. The limitations of intra-CSF chemotherapy include a comparatively small number of agents available with which to treat and limited diffusion into tumors $>2 \mathrm{~mm}$ in diameter [1]. As stated above, there is currently no standard treatment for LM, and treatment options may differ according to the clinical and radiological presentation of the patient. In patients with rapid deterioration in their clinical status, palliative care needs to be discussed. In patients with more stable neurological function, treatment options should be presented. In instances where there are systemic treatment options, both systemic and intra-CSF treatment represents an option in patients with minimal radiographically defined disease.

In recent studies of patients with BC-related LM, the median overall survival rates have varied from 7 weeks to 5 months. One-year survival in BC-related LM varies from 7 to $24 \%$ $[1,4]$. Nonetheless, amongst all solid tumor-related LM, LM secondary to BC is known to have the best prognosis [1]. Studies of BC-related LM suggest histological grade, hormone receptor status and triple negative status correlate with LM survival $[1,4]$. In addition to pathology, the following factors have been found to be associated with overall survival: performance status, age at LM diagnosis, treatment modality, number of prior chemotherapy regimens, concurrent use of systemic and intra-CSF chemotherapy, systemic disease status, and the initial clinical and cytological response to treatment $[1,5]$. According to the National Comprehensive Cancer Network CNS guidelines, primary tumor histology, performance status, bulky radiographic LM disease, the presence of LM-related encephalopathy and the presence of a block of CSF flow are factors that in part determine response to treatment [6]. Our patient initially presented with several good prognostic factors including non-triplenegative BC status, good neurological function, a high performance status and a limited number of prior therapies for systemic disease, and LM was diagnosed as the first site of BC disease recurrence. However, neuraxis MRI revealed multiple large subarachnoid LM nodules in both the posterior fossa (cerebellum) and cauda equina.

The marked improvement in the neurological function and quality of life of our patient was striking and unusual in the management of LM. As stated above, neurological symptoms and signs of LM rarely improve with treatment and in general are not responsive to corticosteroids [2]. We believe that the restoration of neurological function was secondary to systemic bevacizumab plus vinorelbine administration since improvement was observed before the onset of CNS-directed radiation. Moreover, radiotherapy only rarely leads to significant neurological recovery in LM [1]. As a general principle, an earlier diagnosis of LM generally permits recognition of the disease before the occurrence of fixed neurological deficits, thereby permitting more effective preservation of neurological function and quality of life.

Elevated CSF vascular endothelial growth factor (VEGF) levels and CSF/serum VEGF indices have been reported in patients with LM from a variety of solid tumor-related LM. VEGF concentration has also been reported to be correlated with overall survival in LM [7], suggesting that angiogenesis has an etiopathological role in LM [1]. Bevacizumab is a monoclonal antibody that targets the VEGF ligand and inhibits vascular proliferation. At present, there are only preliminary results of using bevacizumab in the treatment of BC-related brain metastases [8-12]. Prolonged responses with neurological progression-free survival times varying from 2.8 to 11 months have been observed. Additional significant positive effects on neurological function have also been observed in case reports $[9,10]$. 
LeRhun et al.: Prolonged Response and Restoration of Functional Independence with Bevacizumab plus Vinorelbine as Third-Line Treatment for Breast Cancer-Related Leptomeningeal Metastases

Similar to the current case report, Vincent et al. [13] reported a 2-year response in a single BC patient with LM treated with bevacizumab and capecitabine. Additionally, there are reports of both the brain and LM responding to capecitabine [14]. Labidi et al. [9] reported on a case in which there was resolution of neurological symptoms and a prolonged response in a BC-related LM patient treated with bevacizumab and paclitaxel. The efficacy of paclitaxel in the CNS, like that of vinorelbine used in the current case, has been less frequently reported, likely due to the limited CNS penetration. This suggests a primary role of bevacizumab in the neurological and radiographic improvement seen in the current case with the radiographically bulky variant of LM. In a pilot study, a total of 15 patients with LM from various primary cancers were treated with intravenous bevacizumab. A significant decrease in CSF VEGF levels was observed, and clinical, imaging and CSF response or disease stabilization was observed in $54-73 \%$ of the patients. The median overall survival was 14 weeks (range, 2-70 weeks, 6 censored) [15]. Prospective studies are currently evaluating bevacizumab in BC-related LM (NCT00924820, NCT01281696).

\section{Conclusion}

In radiographically bulky LM, systemic therapy, radiotherapy or a combination of both is likely more active than intra-CSF therapy only. The combination of bevacizumab and vinorelbine was safe and effective in our patient notwithstanding its use as a third-line LM treatment. Considering the significant improvement in neurological function and the prolonged response of our patient, bevacizumab, alone or in combination with chemotherapy, might be considered in select LM patients with associated subarachnoid nodular disease as defined by MRI.

\section{Acknowledgement}

The authors would like to acknowledge Nathalie Deligny and Thomas Bachelot for their help during the management of the patient.

\section{Disclosure Statement}

E.L.R. and S.T. declare that they have received research funding from Mundipharma and consulted for Roche.

\section{References}

1 Le Rhun E, Taillibert S, Chamberlain MC: Carcinomatous meningitis: leptomeningeal metastases in solid tumors. Surg Neurol Int 2013;4(suppl 4):S265-S288.

-2 Chamberlain M, Soffietti R, Raizer J, Ruda R, Brandsma D, Boogerd W, Taillibert S, Groves MD, Le Rhun E, Junck L, van den Bent M, Wen P, Jaeckle KA: Leptomeningeal metastasis: a Response Assessment in NeuroOncology critical review of endpoints and response criteria of published randomized clinical trials. Neuro Oncol 2014;16:1176-1185.

-3 Le Rhun E, Taillibert S, Zairi F, Pannier D, Boulanger T, Andre C, Cazin JL, Dubois F, Bonneterre, J, Chamberlain MC: Prolonged survival of patients with breast cancer-related leptomeningeal metastases. Anticancer Res 2013;33:2057-2063. 


\section{Case Reports in Oncology}

LeRhun et al: Prolonged Response and Restoration of Functional Independence with Bevacizumab plus Vinorelbine as Third-Line Treatment for Breast Cancer-Related Leptomeningeal Metastases

-4 Abouharb S, Ensor J, Loghin ME, Katz R, Moulder SL, Esteva FJ, Smith B, Valero V, Hortobagyo GN

Melhem-Bertrandt A: Leptomeningeal disease and breast cancer: the importance of tumor subtype. Breast Cancer Res Treat 2014;146:477-486.

5 Chamberlain MC, Johnston SK, Glantz MJ: Neoplastic meningitis-related prognostic significance of the Karnofsky performance status. Arch Neurol 2009;66:74-78.

6 National Comprehensive Cancer Network (NCCN): Clinical Practice Guidelines in Oncology (NCCN Guidelines) - Central Nervous System Cancers. Version I.2014.

http://www.nccn.org/professionals/physician_gls/pdf/cns.pdf (accessed August 21, 2014).

-7 Herrlinger U, Wiendl H, Renninger M, Förschler H, Dichgans J, Weller M: Vascular endothelial growth factor (VEGF) in leptomeningeal metastasis: diagnostic and prognostic value. Br J Cancer 2004;91:219-224.

-8 Berghoff AS, Sax C, Klein M, Furtner J, Dieckmann K, Gatterbauer B, Wildhalm G, Rudas M, Zielinski CC, Bartsch R, Preusser M: Alleviation of brain edema and restoration of functional independence by bevacizumab in brain-metastatic breast cancer: a case report. Breast Care (Basel) 2014;9:134-136.

\9 Labidi SI, Bachelot T, Ray-Coquard I, Mosbaj K, Treilleux I, Fayette J, Favier B, Galy G, Blay JY, Guastalla JP: Bevacizumab and paclitaxel for breast cancer patients with central nervous system metastases: a case series. Clin Breast Cancer 2009;9:118-121.

10 Yamamoto D, Iwase S, Tsubota Y, Sueoka N, Yamamoto C, Kitamura K, Odagiri H, Nagumo Y: Bevacizumab in the treatment of five patients with breast cancer and brain metastases: Japan Breast Cancer Research Network-07 trial. Onco Targets Ther 2012;5:185-189.

11 Lin NU, Gelman RS, Younger WJ, Sohl J, Freedman RA, Sorensen AG, Bullitt E, Harris GJ, Morganstern D, Schneider BP, Krop IE, Winer EP: Phase II trial of carboplatin (C) and bevacizumab (BEV) in patients (pts) with breast cancer brain metastases (BCBM). J Clin Oncol 2013;31(suppl):513.

12 Lu YS, Chen WW, Lin CH, Tseng LM, Yeh DC, Wu PF, Chen BB, Chao TC, Tsai YF, Huang SM, Shih TTF, Cheng AL: Bevacizumab, etoposide, and cisplatin (BEEP) in brain metastases of breast cancer progressing from radiotherapy: results of the first stage of a multicenter phase II study. J Clin Oncol 2012;30(suppl):1079.

13 Vincent A, Lesser G, Brown D, Vern-Gross T, Metheny-Barlow L, Lawrence J, Chan M: Prolonged regression of metastatic leptomeningeal breast cancer that has failed conventional therapy: a case report and review of the literature. J Breast Cancer 2013;16:122-126.

14 Ekenel M, Hormigo AM, Peak S, Deangelis LM, Abrey LE: Capecitabine therapy of central nervous system metastases from breast cancer. J Neurooncol 2007;85:223-227.

15 Groves MD, DeGroot J, Tremont I, Forman A, Kang S, Pei BL, Julie W, Schultz D, Yuan Y, Guha N, Hwu WJ, Papadopoulos N, Camphausen K, Yung WKA: A pilot study of systemically administered bevacizumab in patients with neoplastic meningitis: imaging, clinical, CSF, and biomarker outcomes. Ongoing Clinical Trials. Neuro Oncol 2011;13(suppl 3):iii85-iii91.

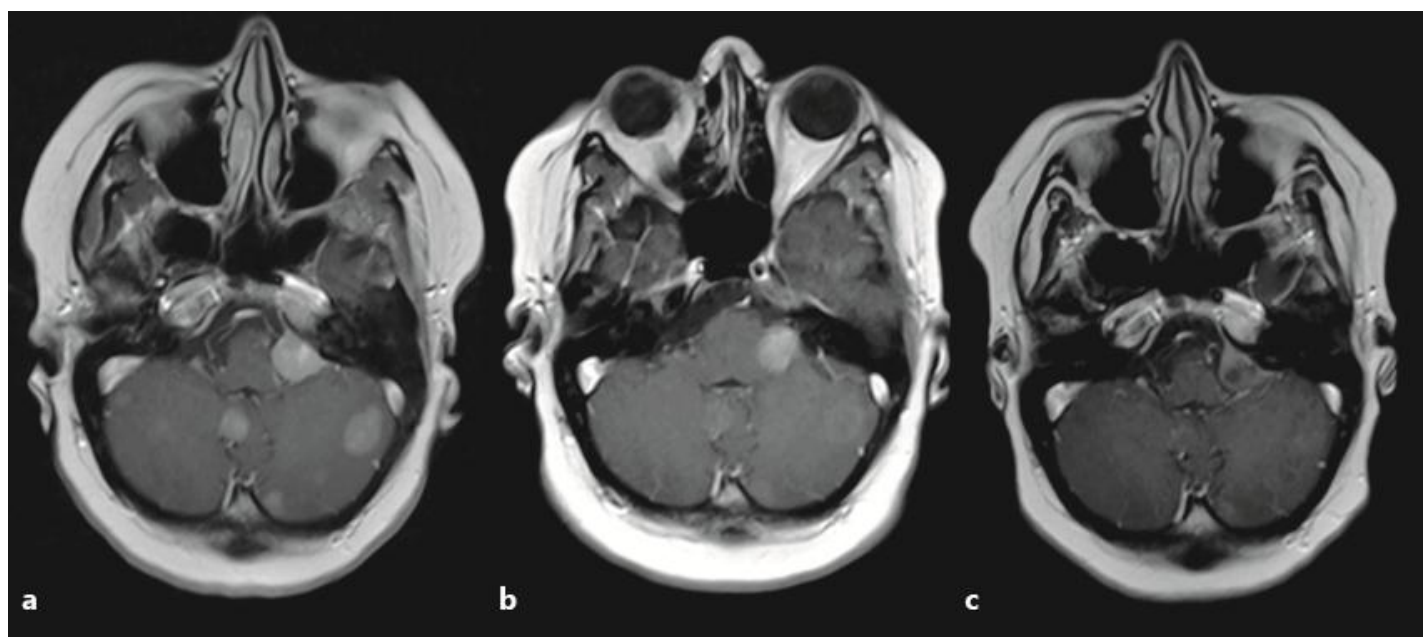

Fig. 1. Brain MRI at baseline (02.05.13; a), after 1 month of treatment with bevacizumab plus vinorelbine at the end of posterior fossa irradiation $(05.06 .13$; b), and after 11.5 months of treatment with bevacizumab plus vinorelbine $(15.04 .14 ; \mathrm{c})$. 